\title{
Docencia en Trabajo Social: potencialidades desde el Trabajo Social agroecológico
}

\author{
Teaching in Social Work: potentialities from the agroecological \\ Social Work
}

Docência em Serviço Social: potencialidades desde o Serviço Social agroecológico

\author{
Sergio Alejandro Díaz Angarita* , Mary Yazmin Fonseca**
}

\section{RESUMEN}

Este artículo exhibe los resultados del trabajo investigativo titulado "Aportes de Actualización Curricular al Curso de Práctica Profesional 'Promoción del Desarrollo Social', del Pregrado en Trabajo Social de la Fundación Universitaria Monserrate. Potencialidades desde el Trabajo Social agroecológico", que demuestra la importancia de la docencia y la investigación para formar un nuevo enfoque para Trabajo Social, tanto para su interacción como para visibilizar y trabajar conjuntamente con comunidades rururbanas, en el fortalecimiento de los procesos de enseñanza y aprendizaje dentro de la práctica profesional de los estudiantes universitarios, rescatando sus experiencias de ese campo y desde el enfoque de Trabajo Social agroecológico.

\begin{abstract}
This article presents the research work results entitled "Contributions to Curricular Updating of the Professional Practice Course 'Promotion of Social Development', of the Undergraduate Degree in Social Work of the Monserrate University Foundation. Potentialities from the agroecological Social Work", which demonstra-
\end{abstract}

Palabras clave: docencia, Trabajo Social, práctica profesional, estudiantes, Trabajo Social agroecológico.

Key

words: teaching, Social Work, professional practice, students,

* Trabajador social, especialista en docencia universitaria. Maestrante en docencia Universidad de La Salle, miembro del Observatorio Ciudadano Juvenil. E-mail: alejandrodiangarita@gmail.com

** Trabajadora Social, especialista en docencia universitaria. Trabajadora Social en la Institución Educativa Soacha para Vivir Mejor, Fe y Alegría. E-mail: Jazzlis@gmail.com 
tes the importance of teaching and research to form a new approach to Social Work, both for its interaction and to make visible and work together with urban communities, in strengthening the processes of teaching and learning within the professional practice of university students, rescuing their experiences in this field and from the agroecological Social Work approach.

\section{RESUMO}

Este artigo apresenta os resultados do trabalho de pesquisa intitulado "Contribuições para a Atualização curricular do estágio profissional 'Promoção do Desenvolvimento Social', do curso de graduação em Serviço Social da Fundação Universitária Monserrate. Potencialidades desde o Serviço Social agroecológico", que mostra a importância da docência e da pesquisa na formação de uma nova abordagem para o Serviço Social, tanto para sua interação como para visibilizar e trabalhar em conjunto com as comunidades rururbanas, no fortalecimento dos processos de ensino e aprendizagem dentro do estágio profissional dos estudantes universitários, resgatando suas experiências neste campo e desde o ponto de vista do Serviço Social agroecológico. agroecological social work

Palavras-chave: docência, Serviço Social, estágio profissional, estudantes, Serviço Social agroecológico. 


\section{Introducción}

La educación superior en Colombia vive actualmente un momento histórico y trascendental para su fortalecimiento y transformación. El cambio más evidente es la virtualización de la educación, adicional a las apuestas dentro del mercado laboral, dando horizontes contundentes para el involucramiento de las instituciones de educación superior, la empresa y el Ministerio de Educación Nacional, con el fin de hacer seguimiento, control de sus ofertas educativas y configurar el mejoramiento de su calidad y el acceso a la misma.

Hablar desde la educación superior es procurar entender las condiciones sociales, económicas y políticas dentro de los territorios inmersos en una nación; de igual manera es conocer los procesos que se adelantan, a nivel territorial, para configurar la educación como el escenario favorable para la construcción de profesionales idóneos al servicio de la sociedad, potenciando de esta forma el enfoque de Trabajo Social agroecológico en la docencia universitaria. El cual consiste "en conocer, trabajar, recopilar, formular e iniciar una propuesta ética, política y metodológica en la construcción y fortalecimiento de las organizaciones sociales que demuestran la importancia de su trabajo desde el sector rural, exaltando su cultura" (Díaz, 2017, p. 136).

La educación superior en Colombia debe enfrentar los retos actuales, tanto en calidad como en accesibilidad, es decir, el cambio de lo presencial a lo virtual, un cambio que se hace necesario para las nuevas generaciones, debido a los avances tecnológicos y a los cambios culturales. Es desde esta óptica que la educación superior debe relacionarse más con los aspectos sociales y con las perspectivas de las comunidades para la construcción de paz y de una sociedad más justa y equitativa. Por ello, podemos mencionar la necesidad de unir esfuerzos entre el sector público y el privado con el fin de fortalecer las condiciones de productividad y así responder a las necesidades ambientales dentro de la educación superior, a los objetivos de desarrollo sostenible en relación con la educación de calidad, a las ciudades y comunidades sostenibles y a las acciones por el clima. Ya que este es un compromiso de todos y sobre todo de aquellos que trabajan por el mejoramiento y calidad de vida de las diferentes comunidades. 
Es importante resaltar que nuestro trabajo se ha desarrollado dentro de la educación superior, reconociendo sus fortalezas y debilidades para, de esta forma, prestar atención y trabajar de forma articulada en la praxis profesional, evaluando las necesidades institucionales como carácter prioritario dentro de los programas de formación y ver el componente de sus asignaturas para generar campos de práctica acordes con estas necesidades. Por ello, se debe analizar las necesidades sociales y sus distintos contextos, para generar propuestas acordes en los campos de práctica profesional.

El propósito de este artículo es mostrar los resultados del trabajo de grado realizado en la Universidad Piloto de Colombia para el año 2019 titulado "Aportes de Actualización Curricular al Curso de Práctica Profesional 'Promoción del Desarrollo Social' del Pregrado en Trabajo Social de la Fundación Universitaria Monserrate. Potencialidades desde el Trabajo Social agroecológico", con el fin de potenciar el enfoque de Trabajo Social agroecológico dentro de la docencia universitaria en Trabajo Social, el cual "trabaja para el fortalecimiento de las organizaciones sociales desde el ámbito cultural desde apuestas éticas, políticas y metodológicas" (Díaz, 2017, p. 136), con el fin de desarrollar prácticas profesionales universitarias dentro de comunidades rururbanas.

\section{Metodología}

Este proceder se desarrolla desde el corte hermenéutico, dado que permite "interpretar, comprender los discursos" (Cordero-Ramos, 2011, p. 89) para comprobar las diferentes posiciones tanto a nivel teórico como normativo. Nuestra investigación es de tipo documental argumentativo, dado que verificamos de esta forma lo que "es correcto o incorrecto, deseable o indeseable y que requiere solución, y llega a una conclusión crítica después de evaluar los datos investigados" (UNID, 2019).

Es decir, la investigación documental argumentativa enseña y revela la forma de analizar la información desde sus diferentes visiones, tanto a nivel teórico como normativo, permitiendo acercarse a examinar y comprender la información a trabajar. El enfoque es cualitativo, dada la relación entre "el diseño, las técnicas, el análisis, y la construcción de teoría” (Scribano, 2008, p. 23), para interpretar la información desde "la recolección y el análisis" (Taylor Bogdan, 2002, p. 158) de los datos. 
Se crearon las siguientes categorías de análisis: a) propósitos del curso, b) contenidos temáticos del curso, c) características de la población y contexto, d) propuesta de la población hacia la práctica.

\section{Resultados}

En primer lugar, se estudió el propósito y el contenido curricular del curso Promoción del Desarrollo Social. El análisis se centró en la pertinencia para la formación de los estudiantes de Trabajo Social de la Fundación Universitaria Monserrate. Dado que en el campo de prácticas profesionales se relacionan los contenidos académicos con la observación del contexto rururbano, se propusieron conceptos nuevos para el fortalecimiento de la asignatura.

En un segundo momento se interpretaron los insumos producidos por los estudiantes de la práctica profesional, para observar su relación y pertinencia entre lo planteado en el microcurrículo del programa de Trabajo Social y la asignatura, ya que cada experiencia profesional desde un campo de práctica específico suma la necesidad de actualización constante de los cursos ofertados, para así atender las necesidades de la población y sus contextos. En este sentido veremos ahora las categorías de análisis.

\section{Propósitos del curso}

El propósito del curso lo ubicamos dentro del objetivo general de su syllabus: "brindar a los y las estudiantes elementos para que apropie los métodos de movilización, y organización social y gerencia social para generar procesos, acciones y actividades que tienen como propósito mejorar las condiciones de vida de la población" (Fundación Universitaria Monserrate, 2019). A partir de este objetivo podemos decir que el proceso de formación está encaminado a la apropiación de métodos y de conocimientos en los estudiantes practicantes, para así permitirles aportar a la movilización de acciones que mejoren la calidad de vida de las poblaciones, desde el trabajo contextual y el fortalecimiento de habilidades y oportunidades comunitarias.

Del mismo modo, los aportes al desarrollo del perfil profesional de los estudiantes los encontramos en el mismo syllabus, catalogados como "aportes al perfil profesional": a) Contribuir al fortalecimiento de la formación intelectual, a través de la promoción de la lectura, análisis 
y discusión de textos académicos; b) Proponer de manera permanente la reflexión sobre el compromiso de la profesión de Trabajo Social con los procesos de cambio en la sociedad por medio de la intervención comunitaria (Fundación Universitaria Monserrate, 2019). Desde el postulado anterior, se afirma que la interacción del profesional de Trabajo Social con los procesos de cambio en la sociedad se da desde una compresión académica, incitada a la reflexión contextual y sumada al compromiso que el estudiante adquiere al momento de interactuar con la población especifica; es decir, tanto los propósitos del curso como sus temáticas académicas están totalmente direccionadas a la construcción de actores profesionales relevantes para la interacción y transformación de un contexto específico, ya que articula elementos que permiten la movilización social y cultural para el fortalecimiento las comunidades base dentro de los territorios.

\section{Contenidos del curso}

El syllabus del curso de Promoción del Desarrollo Social contempla cuatro unidades de trabajo académico: a) Unidad 1. Paradigmas del Desarrollo (Repaso); b) Unidad 2. Concepto de promoción en intervención social; c) Unidad 3. Herramientas para la gestión social y la gerencia social, y d) Unidad 4. Políticas sociales y planes del desarrollo.

En ese mismo sentido, hay una distribución del curso en las siguientes temáticas: a) El desarrollo. Perspectiva histórica; b) Perspectivas alternativas al desarrollo; c) Indicadores del desarrollo; d) Elementos de la promoción del desarrollo; e) Promoción social. Lecturas críticas; f) Gerencia social y Trabajo Social; g) Gerencia social, elementos metodológicos; h) Política social y Trabajo Social; i) Análisis de planes, políticas, programas y proyectos (ejercicio práctico en clase); j) Lo social promocional y su relación con las políticas sociales, y k) Análisis de plan de desarrollo. Finalizando el syllabus se puede analizar su bibliografía, y allí es pertinente resaltar sobre todo la correspondiente a las unidades 3 y 4: a) Esquivel, F. (2005). Gerencia social: un análisis crítico desde el Trabajo Social. Buenos Aires: Espacio; b) Licho, I. (2000). Los instrumentos de la Gerencia Social. La caja de herramientas. En Instituto Interamericano para el Desarrollo Social, Diplomado en Gestión Comunitaria y Gerencia Social. Módulo II. Bogotá; c) Cohen, E. y Franco R. (2005). Gestión Social: Cómo lograr eficiencia e impacto en las po- 
líticas sociales. México: Siglo XXI editores; d) Guadarrama, L. (2006). Política Social y Trabajo Social en el contexto urbano. Revista Cultura y Comunicación, 26-38; e) Observatorio del Caribe Colombiano. (2015). Plan Prospectivo y estratégico de la Región Caribe Colombiana.

Dentro del componente curricular, podemos analizar que la asignatura de Promoción del Desarrollo Social coteja la existencia y la pertinencia entre sus contenidos y sus temáticas, reconociendo la necesidad de su actualización constantemente, dado que se ha avanzado en producción académica, tanto a nivel de Trabajo Social como de otras ciencias humanas que fortalecen la visión de la sociedad y que responden a las necesidades contextuales y culturales de las poblaciones con las que se trabaja.

Para el caso de Soacha, se debe reconocer los avances de organizaciones no gubernamentales en la producción académica, en la construcción territorial, en la importancia del trabajo cultural, y en la participación de hombres y mujeres dentro del territorio, los cuales narran, desde sus distintas visiones, lo aprendido en los procesos sociales y culturales.

Es importante destacar que en cada territorio y en cada práctica profesional se encuentran diferencias y particularidades que demuestran que los territorios pueden asociar las acciones comunitarias como ejes de articulación entre la gestión y la gerencia social, adicional a la reflexión de la participación en la construcción de las políticas públicas que responden a los planes, programas y proyectos de desarrollo comunitario dentro de los territorios.

Asimismo, se debe resaltar la necesidad de avanzar en la construcción pública de lo público, generando una revisión constante de lo existente, lo cual nos permitirá entender el desarrollo y de esta manera aportar nuevos conocimientos dentro del plan del curso.

Trabajar la asignatura de Promoción del Desarrollo Social es aportar a su bibliografía, referenciada en las unidades 3 y 4 , demostrando margen de edades desde el año 2000 hasta 2015 de la Región Caribe, haciendo énfasis en la revisión de su plan prospectivo y estratégico de desarrollo, desconociendo la Región Andina, como los cambios que se han evidenciado en el país en relación a la soberanía alimentaria, el Trabajo Social agroecológico, migración, procesos y acuerdos de paz, 
cuidado y protección de líderes sociales, emprendimiento social y objetivos de desarrollo sostenible, entre otros.

Esta bibliografía referenciada comprende temáticas e información relevante, pero se recomendaría que la selección considere avances, reflexión de nuevas necesidades y desarrollos locales logrados mediante la implementación de la práctica profesional desarrollada desde hace siete años; necesidades concernientes al abordaje socioambiental, agroecológico y a la soberanía alimentaria, lo cual ayudaría a mejorar los procesos sociales que se vienen adelantando desde la Institución Educativa Soacha para Vivir mejor, Fe y Alegría.

Adicionalmente, hay que mencionar que el contexto de la comuna seis del municipio de Soacha ha hecho parte de la construcción del territorio y de la identidad del profesional de prácticas profesionales de Trabajo Social de la Fundación Universitaria Monserrate. Este aporte se ha desarrollado desde la caracterización del contexto, que apoya y genera el seguimiento al plan de desarrollo a nivel local, y la articulación con otras entidades que pertenecen y trabajan por el desarrollo del territorio.

\section{Características de la población y el contexto}

La población de la comuna seis de Soacha Cundinamarca barrio el Altico, pertenece al contexto rururbano del municipio, dada su cercanía a la expansión urbana, tal como lo afirma Sereno \& Santarelli (2012), haciendo contraste por relacionar espacios vacíos con poblados, generando espacios de continuidad/discontinuidad y de concentración/ dispersión, ya que carecen de redes de infraestructura, equipamiento y servicios mínimos que aseguren la calidad de vida de sus población, por apropiación y organización del espacio irregular y complejo (p. 46), entendida desde los estudiantes de octavo semestre de Trabajo Social de la Fundación Universitaria Monserrate, los cuales han desarrollado su práctica profesional en la Institución Educativa Soacha para Vivir Mejor, Fe y Alegría.

Se evidencian necesidades de la población dada la interacción con el medio, tales como: escombreras, explotación de la montaña, huesera, ladrilleras, destrucción del paisaje, consumo y distribución de sustancias psicoactivas e inseguridad. 
Para atender estas problemáticas se vienen adelantado procesos comunitarios desde el accionar de la educación social de los estudiantes practicantes de la Fundación Universitaria Monserrate y la Institución Educativa Soacha para Vivir mejor Fe y Alegría, uniendo esfuerzos con algunas organizaciones sociales del sector, las cuales trabajan en la recuperación del territorio con iniciativas de empoderamiento comunitario desde el Proyecto Comunitario de Siembra y Comercialización de Orellana -hongos-, liderado por mujeres y madres productivas, que permite la consolidación del tejido social dentro de la comuna seis de municipio.

\section{El contexto social e institucional rururbano de Soacha para} la evaluación de la asignatura Promoción del Desarrollo Social Para entender el contexto rururbano del municipio de Soacha, es importante retornar a su memoria histórica, ya que su territorio comprende una dualidad de asentamientos sociales y desplazamientos internos, lo cual es consecuencia de la movilidad interna que ha sufrido Colombia por causa de la violencia interna, hechos que han configurado la identidad poblacional de las personas que lo habitan.

Soacha está ubicado en un territorio ancestral, en el que hubo asentamiento del pueblo Muisca. Herencia de ellos es su nombre, el cual se divide en dos palabras: sua, que significa sol, y cha, que significa varón; es por eso que el municipio de Soacha significa “ciudad del varón del sol". Como afirma Mayorga (2015), este es el punto de partida, en donde podemos conocer la importancia de su patrimonio cultural y saber algunas de las afectaciones que persisten en el territorio, algunas de ellas relacionadas con el medio ambiente (escombreras, huesera, ladrilleras), consumo y distribución de sustancias psicoactivas, población flotante, inseguridad (hurtos, y poca presencia policial) y ausencia estatal-local.

En la actualidad, su patrimonio cultural se contempla desde el Salto del Tequendama, las ruinas de Tuso, la Hacienda Canoas y algunos parques ecoturísticos dentro del municipio, como también el Museo arqueológico Nueva Esperanza, que cuenta con una colección de 300 piezas arqueológicas encontradas en el territorio.

Para ubicarnos contextualmente, es pertinente hablar de su caracterización poblacional y también de su formación territorial. 
Soacha cuenta con 347 barrios, ubicados en seis comunas urbanas y con dos corregimientos rurales. Más del cincuenta por ciento de los asentamientos son ilegales: sin títulos de propiedad y con una pobre inversión pública. Aproximadamente el treinta por ciento de los barrios están ubicados en zonas de alto riesgo natural. Desde la década de los años 80, Soacha presenta una alta tasa de crecimiento; su población es de 398.000 habitantes, según cifras oficiales del DANE, pero la propia Administración Municipal calcula que en realidad corresponde a unas 700.000 personas, de las cuales el 60 por ciento hacen parte de niveles 1 y 2 de la encuesta Sisbén, con lo cual se estima que se trata de población pobre, con escasas prioridades de inversión en políticas sociales. (Corporacion et al., 2010, p. 6)

De lo anterior podemos destacar que Soacha es un municipio en crecimiento continuo, demostrando diferentes problemáticas que afectan el territorio, tanto por la carencia de los servicios básicos, como en la calidad de vida de sus habitantes, sumando nuevas problemáticas, como el desplazamiento de población flotante, tanto nacional como venezolano, y de esta forma se establece rezago social y cultural de la ciudad aledaña que es Bogotá, la capital de Colombia.

\section{Contexto de la red interinstitucional}

Avanzando en nuestro racionamiento, nos adentrarnos en la visión de la Institución Educativa Soacha para Vivir Mejor Fe y Alegría, ya que, desde 2012, cuenta con la modalidad de concesión en conjunto con la administración municipal, para prestar los servicios de institución educativa ubicada en la comuna seis del municipio, situada en la periferia del casco urbano; Fe y Alegría, como institución educativa, es un agente que impulsa procesos de educación popular, promoción social y comunitaria, vinculados al territorio con alianzas interinstitucionales que fortalecen el trabajo en red para contribuir a una acción de mejora para la comunidad interna y externa de la comuna, la cual esta representada en niños, niñas, adolescentes, jóvenes y mujeres, los cuales hacen parte del proceso dentro de la institución educativa.

Para 2018 se realizó, por parte de la institución educativa, el tercer estudio de contexto, el cual se centró en tres ejes de trabajo: caracterización de la población estudiantil, desarrollo comunitario e innovación pedagógica, con el fin de brindar información que aportara al me- 
joramiento de la institución educativa dentro de la comuna seis, y ser así un referente de cambio para la comunidad en general. Este estudio contó tanto con la participación de estudiantes, padres de familia, docentes y líderes de la comunidad, como con integrantes del Servicio de Jesuitas a Refugiados (SJR), Corporación Kairós, Ficonpaz, Asociación Codo a Codo, Casa pastoral Nuestra Señora del Camino, Casa Juvenil Adelaida de Cicé y el Programa de Juventud Generación de Paz de la Alcaldía de Soacha.

Los resultados del estudio arrojaron datos de actualización, tales como la edad, género, nivel de escolaridad, régimen de salud, estrato socioeconómico, tipo de vivienda, ocupación y número de personas que conviven en el núcleo familiar. En su segunda fase, se reflexionó acerca del desarrollo comunitario como aspecto primordial y necesario, que resalta el tema valorativo y evaluativo de una propuesta comunitaria desde la mirada y percepción de los beneficiarios, dado que escuchar sus voces es conocer de antemano qué debemos mejorar continuamente y cómo su participación fortalece todo el proceso. Lo cual es parte del tercer informe contextual del trabajo interinstitucional y barrial que se viene adelantando en Soacha.

Dentro de esta estrategia se evaluaron las acciones comunitarias realizadas por la Institución Soacha para Vivir Mejor Fe y Alegría, la cual resalta como agente de transformación social en la comuna seis de Soacha en el barrio el Altico, y como entidad articuladora para encuentros sociales y de participación. Estas acciones comunitarias se definen en las siguientes cuatros líneas de trabajo; a) Arte y Cultura para la Paz, b) Emprendimiento Comunitario, c) Participación Juvenil, y d) Comunicación Comunitaria.

Arte y cultura para la Paz es todo lo relacionado con expresiones deportivas, manualidades artísticas y danzas para la construcción de paz. Dentro de ellas encontramos deportes como fútbol, baloncesto, manualidades, break-dance, danzas, y música. El Emprendimiento Comunitario se relaciona con la capacitación y formación para el trabajo, es decir, la formación en sistemas y competencias ofimáticas. La Participación Juvenil aborda todo lo relacionado con las nuevas ciudadanías y la construcción de paz, los derechos humanos y la construcción de la posición política juvenil, contando con la participación del Grupo juvenil -participAcción-; y la Comunicación Comunitaria es el trabajo 
relacionado en red desde las emisoras escolares y las emisoras comunitarias para todo el contexto de la comunidad, es decir, se mantiene el trabajo con Xuacha stereo.

\section{Propuesta de la población hacia la práctica}

Los aportes de la población beneficiaria del ejercicio en red que se desarrolla desde la Institución Educativa Soacha para Vivir Mejor Fe y Alegría hacia la práctica profesional de Trabajo Social de la Fundación Universitaria Monserrate, van direccionados hacia el continuo fortalecimiento de los procesos internos en el Centro Educativo, contando con a) mayor interacción de la escuela con la comunidad, b) empoderamiento y desarrollo comunitario, y c) apoyo y acompañamiento a las familias a todos los procesos de participación social, y tienen como finalidad articular los trabajos desarrollados fuera de la institución educativa, para comenzar a mitigar las diferentes problemáticas sociales ya detectadas.

De acuerdo con lo encontrado en el tercer estudio de contexto 2018-2019, la Institución Educativa Soacha Para Vivir Mejor es un líder de procesos de desarrollo comunitario, interviniendo en contextos en los que habitan personas en circunstancias de vulnerabilidad, con el fin de transformar el territorio y su realidad mediante el empoderamiento de niños, niñas y adolescentes del municipio de Soacha, específicamente en la comuna seis desde la Educación Fe y Alegría desde el componente comunitario, aprovechando que es una institución pionera, encargada de fomentar la educación a los sectores vulnerables, y apunta a la educación popular promoviendo los derechos de la niñez, la adolescencia y la participación social de la juventud.

Fe y Alegría, en asociación con Casa Juvenil Adelaida de Cicé, Asociación Codo a Codo, Casa Pastoral Nuestra Señora del Camino, Corporación Kairós, Ficonpaz, SJR Colombia y el programa de Juventud Generación de Paz-Alcaldía de Soacha, se articula para trabajar en torno de la utilización y el buen uso del tiempo libre, con procesos innovadores que ayudan a reducir algunas problemáticas sociales, entre las cuales se encuentran la seguridad zonal, el consumo de sustancias psicoactivas y la divulgación de propuestas comunitarias que ayuden a reducir el trabajo infantil y fortalecer la emisora escolar y comunita- 
ria, con el fin de fortalecer procesos sociales que buscan el desarrollo continuo de la comunidad. Por eso la importancia de realzar la labor desarrollada desde la articulación interinstitucional y los nuevos enfoques que se van adelantando desde la práctica profesional de Trabajo Social, dado que desde el enfoque en Trabajo Social agroecológico se busca fortalecer las organizaciones de base y el trabajo en red, para rescatar la voz cultural de las comunidades, las cuales conocen, viven y socializan dentro de su territorio la territorialidad que los caracteriza. Ya que niños, niñas, adolescentes, jóvenes y mujeres de la comuna seis demuestran que emplean de manera positiva el acompañamiento profesional, y que pueden gestarse nuevos procesos de emprendimiento social y comunitario que beneficien a todos.

\section{Conclusiones y discusiones}

En primer lugar, reconocemos que el nuevo enfoque de Trabajo Social agroecológico se ha venido desarrollando en distintos trabajos investigativos de carácter comunitario, con comunidades campesinas, urbanas y rururbanas. Este enfoque prioriza la reflexión-acción en el contexto, desde el entendimiento y la comprensión de las dinámicas sociales que se desarrollan tanto en comunidades apartadas de las zonas urbanas como en aquellas que crecen en la periferia de las ciudades; observando y acompañando las iniciativas propias de empoderamiento, rescate tradicional y cultural de las mismas organizaciones sociales de base.

Asimismo, este enfoque "posibilita el acercamiento de este a los procesos comunitarios para fomentar estrategias que exaltan la vida cultural de las mismas comunidades y para evidenciar dentro de ellas transformaciones que resisten a los procesos económicos" (Díaz Angarita, 2017, p. 136). Podemos decir que este enfoque agroecológico en Trabajo Social aporta al fortalecimiento de la asignatura de Promoción del Desarrollo Social, desde comprender y entender los cambios sociales que poseen las comunidades para su libre desarrollo y desde iniciativas sociales de emprendimiento y reconocimiento de actores, como en el caso de Soacha, con madres y mujeres productivas bajo el Proyecto Comunitario de Siembra y Comercialización de Orellana -hongos-, para ver la creación de nuevos vínculos, tanto familiares (comunitarios) como laborales, que surgen en el atacar distintas problemáticas. 
Unido a ello, el autor del enfoque de Trabajo Social agroecológico nos invita a "conocer, trabajar, recopilar, formular e iniciar una propuesta ética, política y metodológica en la construcción y fortalecimiento de las organizaciones sociales, exaltando su cultura" (Ibíd), ya que, entendiéndolo, debemos pensarnos la educación universitaria desde la investigación contextual y la praxis del profesional en Trabajo Social, lo cual permite unir conceptos propios de la profesión como de otras ciencias del saber, con el fin de ayudar, mejorar y desarrollar juntamente con las organizaciones sociales y de base, su idiosincrasia, su cultura y el desarrollo de su identidad ante los cambios sociales, políticos y económicos.

En segundo lugar, invitamos tanto a las instituciones de educación superior como a la Fundación Universitaria Monserrate en Colombia para que, desde sus diferentes programas académicos y desde sus diferentes facultades, se incentive a los estudiantes a realizar más procesos investigativos en los diferentes contextos, y al programa de Trabajo Social a que, desde el contexto rururbano, pueda consolidar la experiencia profesional de sistematización de experiencias, dado que realizarla es retroalimentar y aportar al desarrollo profesional del estudiante.

En tercer lugar, podemos dejar abierta la discusión para que podamos visibilizar, desde los contextos comunitarios, la importancia de consolidar grupos de investigación, tanto públicos como privados, para visibilizar e incentivar las iniciativas que exaltan la identidad del territorio, dado que utilizar la visión de niños, niñas y jóvenes de contextos rururbanos puede aportar a la construcción de los territorios y a la construcción de paz, ya que, desde la participación ciudadana, todos pueden compartir, consolidar y construir nuevas formas de entender la realidad y las problemáticas sociales. Cada territorio puede ser visibilizado desde su cotidianidad y desde su organización, para ser escuchado y atendido por las autoridades municipales, departamentales y nacionales. Porque tanto la educación como la docencia son el camino para mejorar las distintas comunidades que sufren problemáticas sociales.

Finalmente, hacemos recomendamos el fortalecimiento de la asignatura Promoción del Desarrollo Social desde el enfoque de Trabajo Social agroecológico, la cual vincula conocimientos agroecológicos, ecológicos, antropológicos, geográficos y sociológicos, y da la visión de 
sostenibilidad para trabajar con diferentes comunidades rurales, urbanas y rururbanas del territorio nacional.

\section{Bibliografía}

Cordero-Ramos, N. (2011). Trabajo Social y Hermenéutica Crítica: una opción metodológica para desvelar elementos éticos en los orígenes de la profesión en Sevilla. Portularia, XI(1), 87-97.

Corporación Infancia y Desarrollo (CID), Fundación Menonita Colombiana para el Desarrollo (Mencoldes), Fundación Servicio Jesuita a Refugiado (SJR), Fundación para la Educación y el Desarrollo (FEDES), Personería Municipal de Soacha, Pastoral Social de So. (julio 2010). Un Silencio que Grita este Municipio. Soacha: Reel Ridden.

Díaz Angarita S. A. (2017). Campesinado, agricultura familiar y un Trabajo social agroecologico. Catedra Paralela No 14. Colegio de Profesionales de Trabajo Social Segunda circunscripción, Santa Fe. Escuela de Trabajo Social Facultad de Ciencias Políticas y RRII, UNR. Rosario, Argentina.

Diaz, A. S. A. \& Fonseca, M. Y. (2019). Aportes de Actualización Curricular al Curso de Prácticas profesional "Promoción del Desarrollo Social" del Pregrado en Trabajo Social de la Fundación Universitaria Monserrate. Potencialidades del Trabajo Social agroecológico. Bogotá, Colombia: Universidad Piloto de Colombia.

Fundación Universitaria Monserrate. (21 de enero de 2019). Syllabus Promoción del Desarrollo Social. Información curricular de un curso del programa de Trabajo Social. Bogotá D.C.

Mayorga, G. M (2015) Realidades territoriales de Soacha, analisis de la vida cotidiana. Investigación del Programa de Psicologia de la Corporación Universitaria Minuto de Dios Regional Soacha. Soacha, Cundinamarca: Corporación Universitaria Minuto de Dios Regional Soacha.

Scribano, A. O. (2008). El proceso de investigación social cualitativo. Buenos Aires: Prometeo libros.

Sereno. C. A \& Santarelli Serer S. A. (2012). El rururbano: un espacio de vulnerabilidad y riesgo. Estudio cualitativo en la ciudad de Bahía Blanca, provincia de Buenos Aires, Argentina. Cuadernos de Geografía: Revista Colombiana de Geografía, 21(2), 149. DOI: https://doi.org/10.15446/rcdg.v21n2.28263. 
Taylor Bogdan, S. J. (2002). Introducción a los métodos cualitativos de investigación. Barcelona: Paidós.

UNID. (27 de julio de 2019). Repositorio de Recursos Digitales. Recuperado de Repositorio de Recursos Digitales: http://brd.unid. edu.mx/recursos/Metodologia_de_la_Investigacion/MI08/ Tecnicas_de_investigacion.pdf?603f00. 